ENCYCLOPEDDE Encyclopédie berbère

BERBERE

$19 \mid 1998$

19 | Filage - Gastel

\title{
Flissa / Iflissen
}

E.B.

\section{OpenEdition}

Journals

Édition électronique

URL : http://journals.openedition.org/encyclopedieberbere/1944

DOI : 10.4000/encyclopedieberbere.1944

ISSN : 2262-7197

\section{Éditeur}

Peeters Publishers

\section{Édition imprimée}

Date de publication : 1 février 1998

Pagination : 2857-2862

ISBN : 2-85744-994-1

ISSN : 1015-7344

\section{Référence électronique}

E.B., «Flissa / Iflissen », Encyclopédie berbère [En ligne], 19 | 1998, document F30, mis en ligne le 01 juin 2011, consulté le 25 septembre 2020. URL : http://journals.openedition.org/encyclopedieberbere/ 1944 ; DOI : https://doi.org/10.4000/encyclopedieberbere.1944

Ce document a été généré automatiquement le 25 septembre 2020

(c) Tous droits réservés 


\section{Flissa / Iflissen}

E.B.

Tribu de grande Kabylie occupant le littoral entre Dellys et Azzefoun (ex PortGueydon), rendue célèbre par la fabrication de sabres auxquels elle donna son nom. Il s'agit plus précisément des Iflissen l-Bahr (les Iflissen de la mer) qui doivent leur nom à leur implantation dans la Kabylie maritime. Le nom de Flissa / Iflissen a été rapproché de celui des Isaflenses*, puissante gens (tribu) de l'Antiquité qui est connue pour avoir pris part dans les années 373-375à la très grave révolte de Firmus*. Seuls une vague analogie entre les deux noms et le fait que les Isaflenses occupaient peut-être le bassin de Tisser peuvent être présentés en faveur de cette identification défendue par Berbrugger, Carette, Cat et Boulifa mais rejetée par S. Gsell, C. Courtois et J. Desanges. Il est bon, toutefois de noter, à la suite de Carette, qu'une fraction, celle des "Flisset Melil" était établie sur les bords de l'oued Isser et donc occupait le territoire qu'on prête volontiers aux Isaflenses. Vers l'intérieur les limites du territoire des Iflissen l'Bafir correspondent, au sud, à la montagne de Dra Moulit par où passe la ligne de partage des eaux entre le bassin du Sébaou et le versant méditerranéen.

2 Cette Kabylie littorale est bordée d'une côte peu accueillante, accidentée et semée d'écueils. Les deux ports ayant quelque importance sont situés aux deux extrémités du territoire: Dellys (l'antique Rusucuru) et Azzefoun (Rusazus), mais pour le commerce international ce sont, à l'extérieur, les ports d'Alger et de Bejaïa qui assurent les fonctions essentielles. Le sol n'est pas plus favorable que la mer, une agriculture pauvre ne produit que des figues, du raisin de treille et de l'orge. Cependant les premiers visiteurs, en particulier Carette, remarquent que la pauvreté des terres ne correspond pas à l'aspect de l'habitat qui est assez cossu; les maisons sont construites en pierres, sont pourvues d'un toit en tuiles et possèdent souvent un étage. Cette relative richesse s'expliquerait par la spécialisation artisanale des Flissa, la fabrication d'armes blanches réputées. 


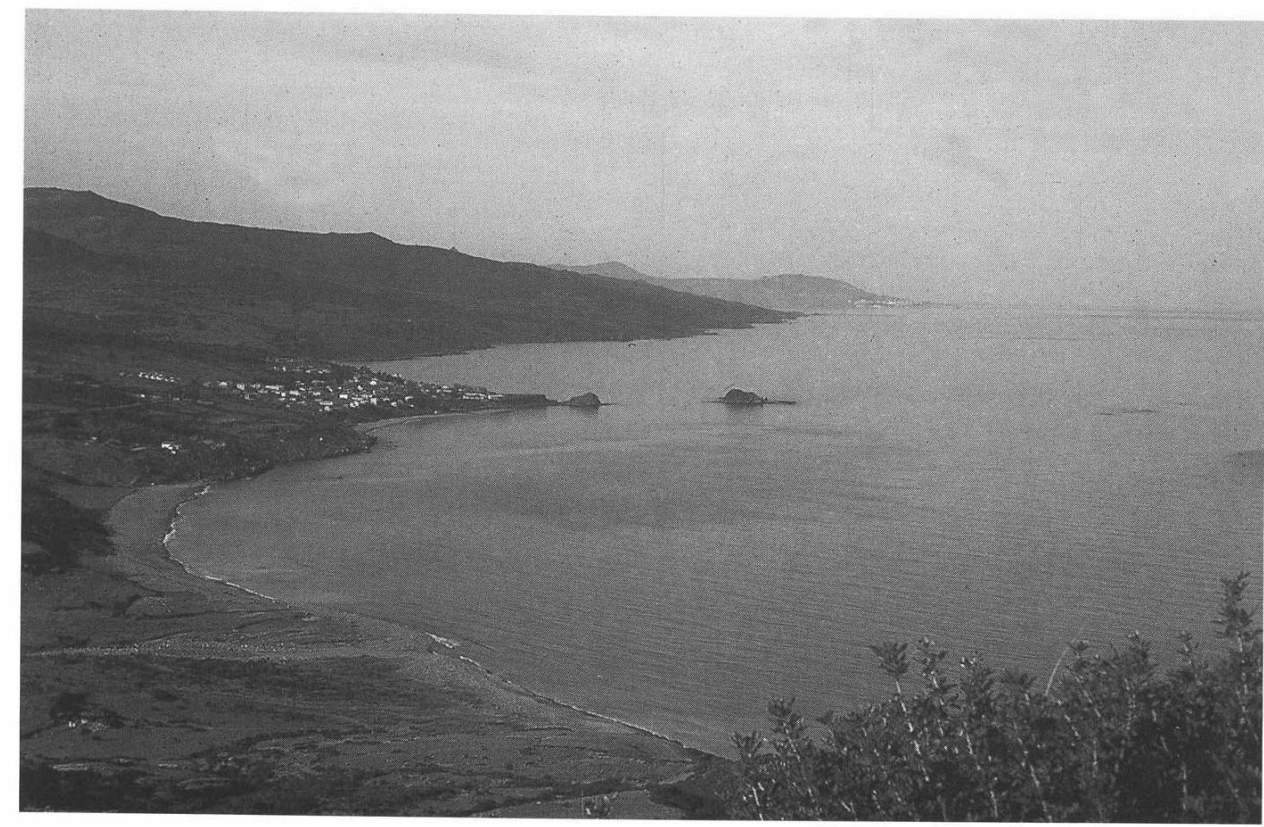

Bien que les auteurs, Devaux, Farine, Letourneux, Maunier etc. insistent sur le fait que plusieurs tribus kabyles pratiquaient la métallurgie et particulièrement la fabrication d'armes blanches, seuls les Flissa acquirent une renommée suffisante pour que leur nom restât attaché à cette spécialité. Bien mieux, "flissa" est l'un des rares mots kabyles à être passé dans la langue française. Il figure dans le dictionnaire Larousse universel, en deux volumes (1922), sous la description suivante : «Flissa, grand couteau kabyle à lame longue asymétrique, presque droite à un seul tranchant et à pointe aiguë ", cette notice est même accompagnée d'une illustration. L'édition de 1930 du Larousse $d u x^{e}$ siècle en six volumes complète en donnant des dimensions et une curieuse précision sur la poignée qui "a la forme d'un fémur de mouton". D'après $\mathrm{Ch}$. Farine (1882), les Barbachas fabriquaient eux aussi des sabres, des couteaux et différents instruments agricoles : socs d'araires et pioches principalement. De même, les Beni Yenni, aujourd'hui spécialisés dans la seule orfèvrerie émaillée, étaient aussi reconnus, au XIX ${ }^{\mathrm{e}}$ siècle, comme des armuriers réputés.

4 Les flissa ou sabres kabyles ont fait l'objet d'une étude très minutieuse et fort complète de la part de C. Lacoste (1958) qui reconnaît plusieurs catégories mais ces armes ont toutes pour trait commun de ne posséder qu'un seul tranchant, ce qui permet de les classer dans la famille des sabres.

5 La première catégorie comprend les armes de type courbe dont le tranchant dessine une ligne parallèle au dos, la largeur de la lame est donc constante et la pointe peu développée, aussi ces armes de type courbe étaient utilisées de taille. Elles ne se distinguent guère des yatagans, armes d'origine orientale d'abord importées en Kabylie puis reproduites sur place.

6 La catégorie des armes droites comprend trois types fondés sur la longueur de la lame qui varie de 110 à $41 \mathrm{~cm}$. Dans les grands sabres droits la lame, qui peut dépasser un mètre de longueur, pour une largeur de 30 à $38 \mathrm{~mm}$, a un tranchant à double courbure opposé à un dos rectiligne, de sorte que la plus grande largeur de la lame se situe dans 
la partie médiane ; au-delà, la lame est rétrécie, dégageant une pointe très effilée d'une longueur de 35 à $50 \mathrm{~cm}$. Selon $\mathrm{C}$. Lacoste, une telle lame réunit deux parties fonctionnelles : la région proximale, qui correspond aux trois quarts de la longueur, est destinée à frapper de taille, la partie distale, qui correspond à la pointe, doit servir aux coups d'estoc. Ces grandes armes à lame droite sont typiques de la Kabylie, elles seules ont droit au nom de flissa.

7 Les armes droites de petites dimensions (petits sabres et couteaux) ont à peu près la même silhouette et continuent d'être fabriqués pour être vendus aux touristes.

Entre le type courbe qui appartient à la famille des yatagans et le type "grand droit" qui est le vrai flissa, il existe plusieurs intermédiaires dont la lame est moins effilée ou dont la largeur est à peu près constante ou, au contraire, présente une double courbure.

9 La poignée, toujours dépourvue de garde, est faite de deux plaquettes de bois qui prolongent la fusée en fer massif ; l'ensemble est recouvert de cuir ou d'un plaquage de cuivre. La poignée se termine par un pommeau de bois sculpté qui, à l'origine, devait représenter une tête de chien. Sur certaines armes de type yatagan, le pommeau est à ailerons, les deux plaques de cuivre qui constituent la poignée s'évasent à l'extrémité en s'écartant de la fusée.

Les fourreaux sont toujours en bois, constitués de deux valves qui portent chacune, en creux, une empreinte de la face de la lame. Les fourreaux sont faits dans un bois dur, mais les armes les plus précieuses possèdent un fourreau entièrement gainé de cuir ou recouvert de plaques de cuivre. Seule la face externe du fourreau porte un décor gravé, sculpté ou en champlevé.

11 Les lames des flissa portent aussi un décor, il est issu de deux techniques : la gravure du fer et l'incrustation de parcelles ou de fils de cuivre. Ce décor de style géométrique triangulaire, orne les bordures du dos et des contre-tranchant. Il rejoint, par le choix de ses motifs, les principes de l'art décoratif berbère.
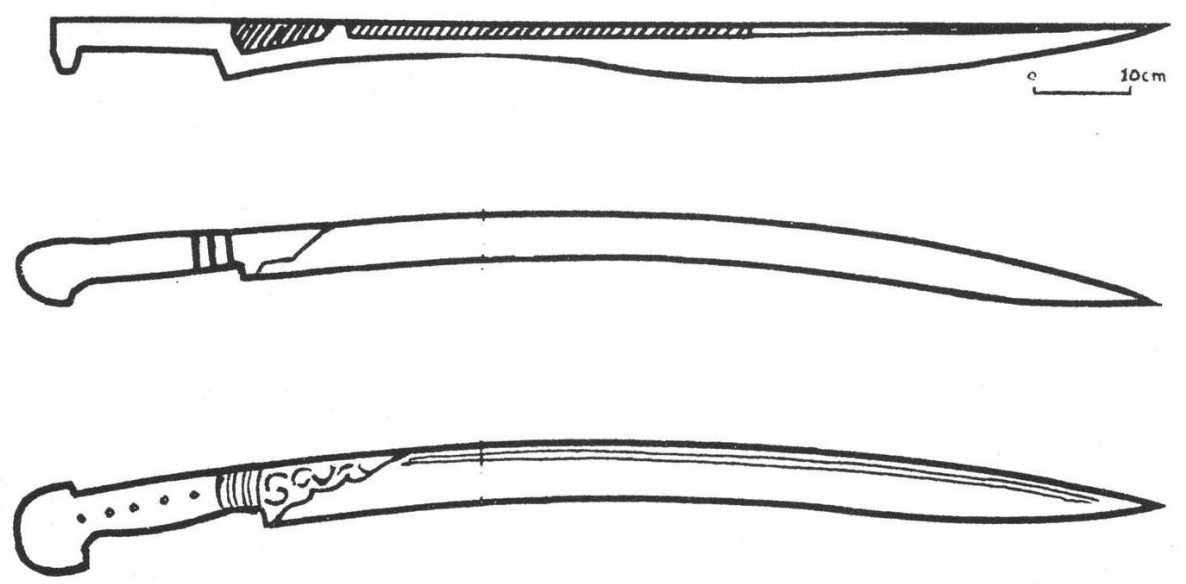

En haut, sabre droit kabyle (flissa), au centre grand sable courbe kabyle (Atayan), dérivé du yatagan balkanique (d'après C. Lacoste-Dujardin). 
Décor d'un flissa ; en haut décors de bordure de dos (d'après C. Lacoste-Dujardin).
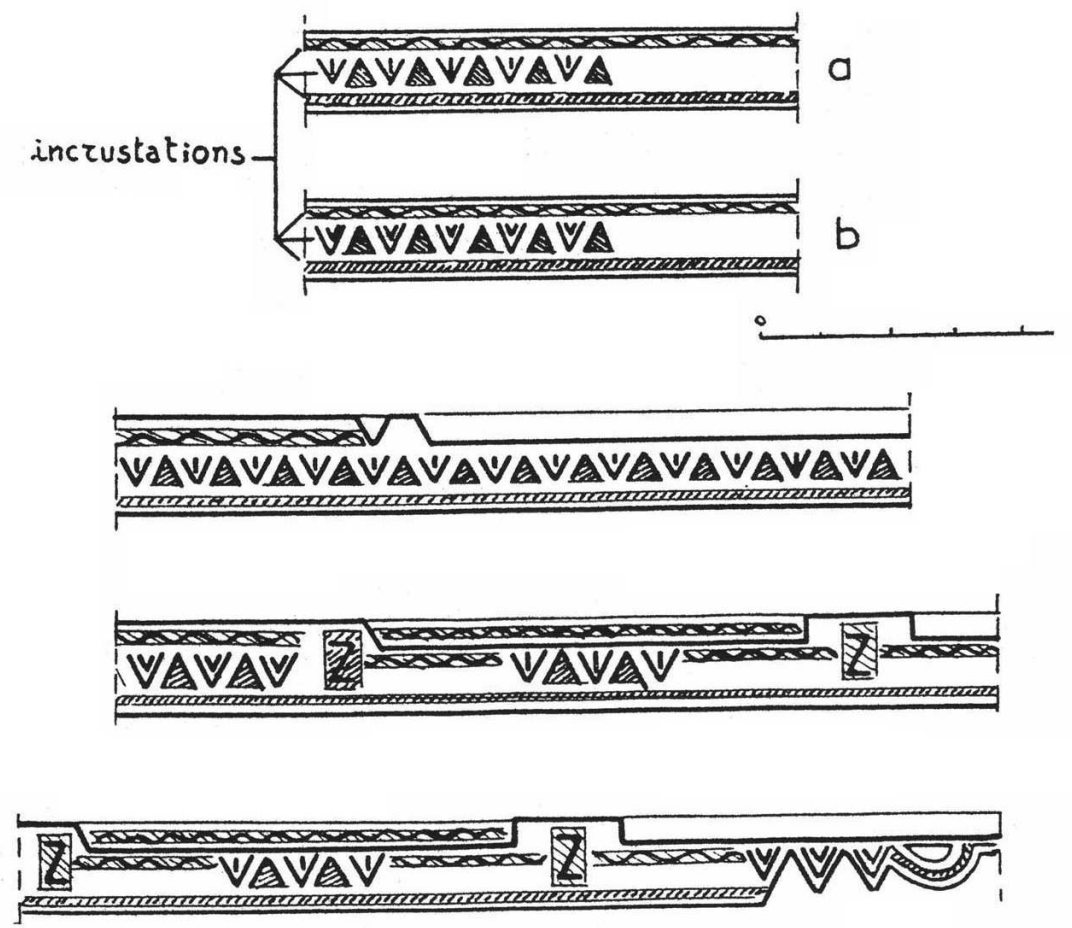

Décor de la fusée et du pommeau d'un flissa : a- type traditionnel ; b- type curviligne.

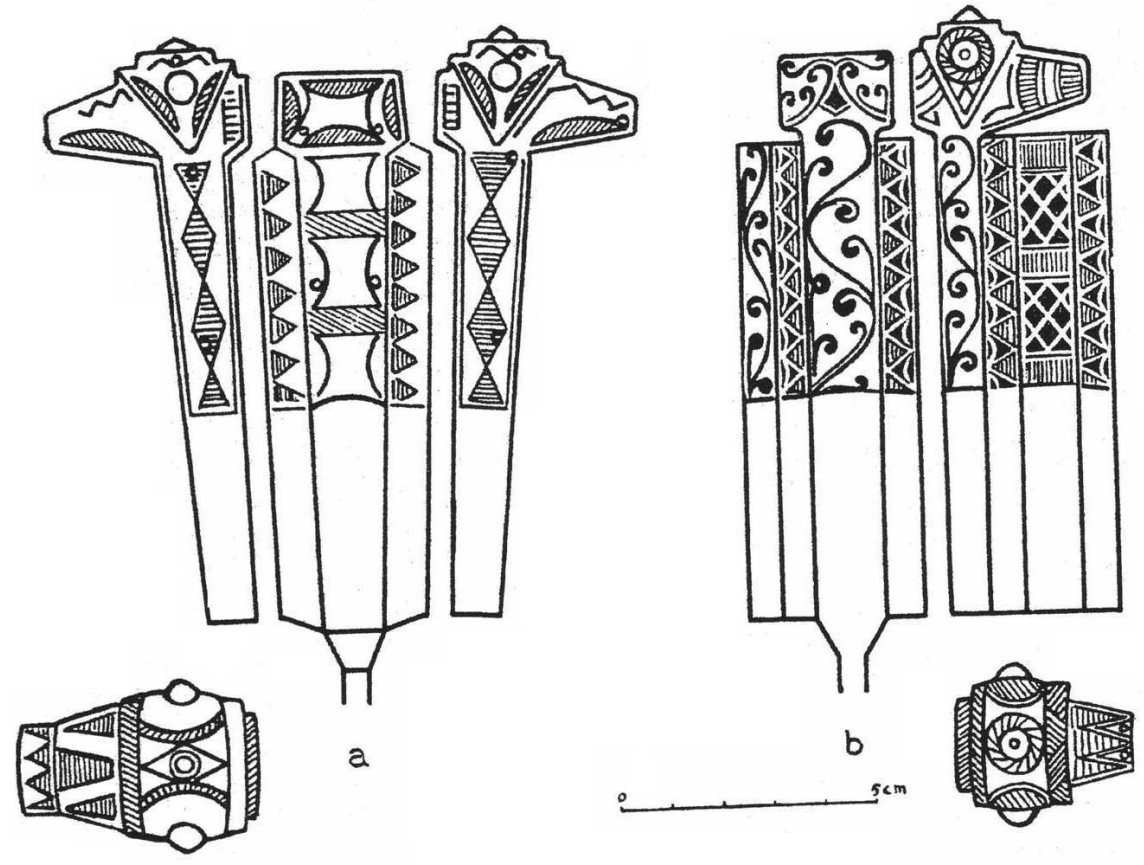

12 Les flissa, le mot et la chose, ont une histoire en grande partie obscure. Le nom est d'apparition récente et d'origine française. Le sabre, en kabyle, n'est pas désigné par le nom de la tribu qui le fabrique, mais par le lieu de provenance des lames (ainsi ajenoui : de Gènes). Les Kabyles peuvent aussi donner un nom dérivé du vocable étranger (ainsi yatagan donna uturam). Les premiers auteurs français qui s'intéressèrent à la Kabylie au 
cours des trois décennies qui suivirent la prise d'Alger furent les propagateurs de la dénomination "flissa".

Mais les Iflissen 1-Bahr ne furent pas les seuls artisans armuriers de Kabylie. Chez les Beni Yenni, il existe une vieille tradition du travail des métaux, aujourd'hui celle-ci est réduite à l'orfèvrerie émaillée, alors qu'en 1925, R. Maunier décrivait encore une sorte de manufacture à Taourirt Mimoun où on fabriquait des "sabres dits flissa" destinés aux touristes. Quarante ans plus tôt, F. Drouet accusait ces mêmes Beni Yenni de copier les armes des Flissa 1-Bahr. On reconnaît leur contrefaçon à la poignée dont il n'arrivaient pas à reproduire la "tête de chimère". En remontant encore dans le temps, nous trouvons le témoignage de Hun (1860) qui cite les productions des Beni Yenni : platines de fusil, " petits yatagans de forme effilée et pointue, appelés agenoui ». Au même moment, Ch. Devaux dans sa monographie sur "Les Kebailes du Djerdjera" cite parmi les produits du pays, les flissa fabriqués dans la tribu des Flisset-Behar (sic) et précise que les forgerons sont nombreux en Kabylie. Quelques années plus tôt, $\mathrm{H}$. Fournel, et antérieurement E. Daumas confirment que la dénomination flissa est d'origine française et désigne les sabres fabriqués par la tribu du même nom : « La tribu des Flissa confectionne des sabres auxquels nous avons donné son nom. »

Les mêmes auteurs et d'autres voyageurs contemporains citent d'autres tribus dans le territoire desquelles se trouvent des mines de fer alors que les Iflissen en sont dépourvus. L'exploitation la plus importante se trouve chez les Beni Soliman et surtout les Barbouchas dont le commerce du fer est la principale source de richesse. Les Iflissen s'approvisionnent en fer chez eux, mais aussi sur les marchés de Béjaïa et d'Alger en fer et acier européens.

Pendant des siècles, au moins à partir du Xvi ${ }^{e}$ siècle époque à laquelle les Turcs firent connaître le yatagan, le flissa fut l'arme principale du kabyle. Capable de frapper de taille aussi bien que d'estoc grâce à sa longue pointe effilée, il était, aux dires des militaires, une arme terrible et convenant particulièrement à la guerre d'embuscades. Le flissa disparut devant la multiplication tardive des armes à feu et, dans la psychologie du guerrier kabyle, ce sabre spécifique de son ethnie céda la place au fusil qui devint l'arme noble que se devait de posséder tout chef de famille. Les villages des Aït Zaoua, qui constituent le cœur du pays flissa, furent longtemps le "Saint-Etienne kabyle" (propos recueilli par C. Lacoste de la bouche d'un ancien armurier). Ces villages ne comptent plus un seul armurier aujourd'hui. La production des flissas cessa peu à peu ; si elle ne disparut pas complètement ce fut en raison de l'intérêt manifesté pour cette arme par de rares touristes et collectionneurs. Les armes authentiques ayant disparu du marché, de petits ateliers reproduisirent des flissas destinés à la vente étrangère, surtout de petits modèles, en mauvais fer et de finition médiocre. Le terrible sabre kabyle était devenu un coupe-papier. Puis même ce dérivé disparut de la production artisanale. Il est remarquable que la personne qui étudia avec le plus de perspicacité les sabres kabyles, publia, en 1976, une monographie sociologique sur les villages flissa d'Ait Zouaou ne fait allusion qu'en une seule ligne à ce produit d'un artisanat disparu. 
Couteau de pacotille et son foureau (photo G. Camps).

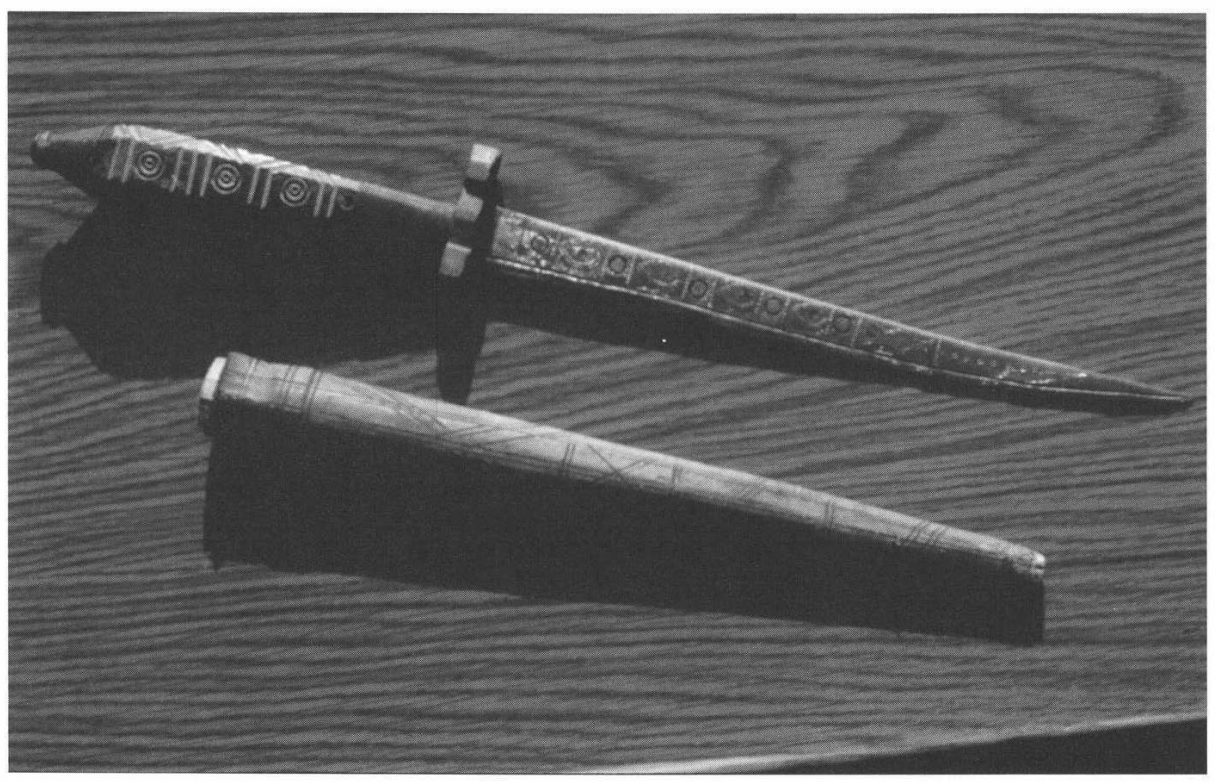

\section{BIBLIOGRAPHIE}

LACOSTE C, "Sabres kabyles", Journal de la Société des Africanistes, t. XXVIII, 1958, p. 111-191

(Bibliographie complète sur la question)

LACOSTE-DUJARDIN C., Un village algérien, structure et évolution récente, Alger, 1976.

\section{INDEX}

Mots-clés : Kabyle, Tribu 